Dinámicas e integración de los mercados financieros de los países del TLCAN

Javier Emmanuel Anguiano-Pita y Antonio Ruiz-Porras 
Lecturas de Economía, 92 (enero-junio 2020), pp. 67-100

Javier Emmanuel Anguiano-Pita y Antonio Ruiz-Porras

\section{Dinámicas e integración de los mercados financieros de los países del TLCAN}

Resumen: El objetivo de este artículo es estudiar las dinámicas del proceso de integración de los mercados de valores gubernamentales, interbancarios, cambiarios y bursátiles de las economías del TLCAN. Para tal propósito, se emplea el modelo generalizado de factores comunes propuesto por Forni, Hallin, Lippi y Reichlin (2005) y series representativas de los rendimientos de los mercados analizados para el período comprendido entre enero de 1995 y diciembre de 2017. Los principales resultados sugieren que: 1) existen asimetrías en el tamaño de los mercados, 2) hay evidencia de cambios estructurales, 3) existen factores comunes entre los mercados financieros, 4) los mercados tienen niveles de integración diferenciados, y 5) los mercados cambiarios y bursátiles son los más sensibles a los componentes comunes. Estos hallazgos pueden ser útiles para analizar la evolución del TLCAN y para proponer políticas económicas y financieras regionales.

Palabras clave: integración financiera; mercados monetarios; mercados cambiarios; mercados interbancarios; mercados bursátiles; modelo generalizado de factores dinámicos; TLCAN.

Clasificación JEL: C58; F36; G15.

\section{Market dynamics and integration of the financial markets of the NAFTA countries}

Abstract: The aim of this paper is to study the dynamics of the integration process of the bond, interbank, currency
and stock markets of the NAFTA region. For this purpose, we use the generalized dynamic factor model originally
proposed by Forni, Hallin, Lippi and Reichlin (2005) and representative series of monthly returns of the analyzed
markets for the period from January 1995 to December 2017. The main results suggest that: 1) There are asymmetries
in the size of the markets; 2) there is evidence of structural breaks; 3) common factors exist among the financial markets;
4) the markets have differentiated levels of integration; and 5) the currency and stock markets are the most sensitive to
the common components. These findings may be useful to analyze the evolution of NAFTA and to propose economic
and financial regional policies.

Keywords: financial integration; monetary markets; currency markets; interbank markets; stock markets; generalized dynamic factor model, NAFTA.

JEL Classification: C58; F36; G15.

\section{Dynamique et intégration des marchés financiers des pays de l'ALENA}

Résumé: L'objectif de cet article est d'etudier la dynamique du processus d'intégration des marchés publics, interbancaires, monétaires et boursiers des économies faisant partie de l'ALENA. À cet effet, nous utilisons le modèle de facteurs communs généralisés proposé par Forni, Hallin, Lippi et Reichlin (2005) et nous analysons une série représentative des rendements des marchés pour la période entre janvier 1995 et décembre 2017. Les principaux résultats suggèrent que: 1) il y a des asymétries dans la taille des marchés, 2) il y a des changements structurels, 3) il existe des facteurs communs entre les marchés financiers, 4) les marchés ont des niveaux d'intégration différenciés et 5) les marchés monétaires et boursiers sont les plus sensibles aux composants courants. Ces résultats peuvent être utiles pour analyser l'évolution de l'ALENA, afin de proposer des politiques économiques et financières régionales.

Mots clés: intégration financière; marchés monétaires; marchés des changes; marchés interbancaires; marchés boursiers; modèle de facteur dynamique généralisé; ALENA.

Classification JEL: C58; F36; G15. 


\title{
Dinámicas e integración de los mercados financieros de los países del TLCAN
}

\author{
Javier Emmanuel Anguiano-Pita $\mathbb{( 1 0}^{\mathrm{a}}$ y Antonio Ruiz-Porras $\mathbb{(}^{\mathrm{b}}$
}

\begin{abstract}
-Introducción. -I. Características de los mercados financieros del TLCAN. -II. Revisión de la literatura. -III. Metodología de análisis. -IV. Base de datos y estadísticas descriptivas. -V. Modelación y análisis econométrico. -Conclusiones. -Anexos. -Agradecimientos. -Referencias.
\end{abstract}

doi: 10.17533/udea.le.n92a03

Primera versión recibida el 27 de febrero de 2018; versión final aceptada el 22 de julio de 2019

\section{Introducción}

El Tratado de Libre Comercio de América del Norte (TLCAN) se considera un acuerdo representativo de la integración económica a nivel mundial. Formalmente, el acuerdo plantea el establecimiento de una zona de libre comercio, la apertura y protección de los flujos financieros - principalmente de Inversión Extranjera Directa - y, en cierta medida, el establecimiento de un marco legal y regulatorio común entre los gobiernos de Estados Unidos, Canadá y México. La principal característica que distingue al acuerdo es que este se encuentra conformado por países con distintos niveles de desarrollo económico y financiero.

a Javier Emmanuel Anguiano Pita: estudiante del Doctorado en Estudios Económicos, Centro Universitario de Ciencias Económico-Administrativas, Universidad de Guadalajara, Zapopan, México. Dirección postal: Periférico Norte $N^{\circ}$ 799, Núcleo Universitario Los Belenes, C. P. 45100. Dirección electrónica: je.ptt@hotmail.com

https://orcid.org/0000-0002-3902-534X

b Antonio Ruiz Porras: profesor investigador titular C y coordinador del Doctorado en Estudios Económicos, Centro Universitario de Ciencias Económico-Administrativas, Universidad de Guadalajara, Zapopan, México. Dirección postal: Periférico Norte N ${ }^{\circ} 799$, Núcleo Universitario Los Belenes, C. P. 45100. Dirección electrónica: antoniop@cucea.udg.mx https://orcid.org/0000-0003-4184-0850 
Anguiano-Pita y Ruiz-Porras: Dinámicas e integración de los mercados financieros...

En este contexto, a más de veinte años de haber entrado en vigor el TLCAN, el cual formalizó la apertura de los mercados de esa región, el desarrollo de estudios que evalúen el nivel de integración en el ámbito financiero continúa siendo relevante. Dentro de la literatura especializada la mayoría de los trabajos sobre la integración de los mercados financieros han analizado los mercados bursátiles, pero, debido a limitaciones metodológicas y de disponibilidad de series de datos comparables se han realizado pocos estudios que involucren otros tipos de mercados.

En este artículo se analiza de manera conjunta la dinámica e integración de los mercados de tasas de interés, tipos de cambio y activos bursátiles pertenecientes a los tres países que conforman el TLCAN. El estudio de las dinámicas e interrelaciones de los mercados es necesario para entender los procesos de ahorro, inversión y expectativas de crecimiento económico en la región. La razón principal de incorporar diferentes tipos de mercados financieros se debe a que las instituciones, el acceso a los sistemas de pagos, los requerimientos de transformación de activos, los riesgos y los problemas de información son múltiples y diversos en los países analizados y entre ellos.

Existen distintos enfoques teóricos que explican las interrelaciones entre los mercados financieros de economías abiertas. Los enfoques más utilizados en la literatura empírica son las condiciones internacionales de paridad, el enfoque de balanza de pagos y el enfoque de mercado de activos (Eiteman, Stonehill \& Moffett, 2016, p. 262). Estos enfoques postulan relaciones diferentes entre los mercados, debido a que, según enfatizan, las dinámicas de los mercados responden a diferentes factores. Por lo tanto, no existen consensos sobre la naturaleza de las interrelaciones ni de las dinámicas de los mercados.

En términos metodológicos, esta investigación se sustenta en la estimación del modelo econométrico de factores dinámicos comunes, siguiendo el enfoque generalizado propuesto por Forni, Hallin, Lippi y Reichlin (2005). El enfoque empleado permite representar un conjunto de series de tiempo mediante su descomposición en su componente común e idiosincrático. Particularmente, aquí se interpretan los factores que explican la dinámica de los componentes comunes como variables latentes que capturan 
las dinámicas de integración entre los mercados. Usar este enfoque no requiere imponer una forma funcional específica sobre el modelo y permite aprovechar la información contenida en las series observadas para capturar los movimientos coincidentes de corto y largo plazo.

Con la finalidad de caracterizar la dinámica de los mercados analizados se utilizan estadísticas descriptivas, pruebas de raíces unitarias y estacionariedad, de cambio estructural endógeno y los criterios de información propuestos por Alessi, Barigozzi y Capasso (2010) y Hallin y Liska (2007). La muestra analizada incluye series mensuales de tasas de interés, tipos de cambio spot e índices de precios bursátiles representativos de los mercados de Estados Unidos, Canadá y México para el período comprendido entre enero de 1995 y diciembre de 2017.

Para evaluar el grado de integración entre mercados se emplea un enfoque similar al utilizado en Forni, Hallin, Lippi y Reichlin (2000), el cual consiste en calcular el porcentaje de la varianza total explicada por el componente común asociado a cada variable observada. Este porcentaje es interpretado como una medida aproximada del nivel de integración entre los mercados de la región. Adicionalmente, se calculan los promedios de los porcentajes agrupándolos por tipo de mercado y por país, con la finalidad de hacer comparaciones sobre el grado de integración financiera en diferentes ámbitos.

Este estudio hace contribuciones econométricas y analíticas a la literatura empírica que estudia el proceso de integración entre los mercados financieros del TLCAN. La contribución analítica de este estudio consiste en extender la investigación sobre la dinámica de la integración financiera en la región y profundizar en el estudio conjunto de distintos mercados financieros.

El artículo se organiza en siete secciones. La sección I presenta una breve caracterización estadística de los mercados estudiados. La sección II incluye la revisión de la literatura. La sección III presenta la metodología empleada en el análisis empírico. La sección IV describe la base de datos e incluye las pruebas estadísticas de raíces unitarias, estacionariedad y cambios estructurales. La sección $\mathrm{V}$ presenta los resultados de la modelación y el análisis econométrico del modelo de factores dinámicos. La última sección sintetiza los principales hallazgos y las conclusiones del estudio. 
Anguiano-Pita y Ruiz-Porras: Dinámicas e integración de los mercados financieros...

\section{Características de los mercados financieros del TLCAN}

Si bien es cierto que los mercados financieros de México han exhibido un rápido crecimiento a partir de su liberalización en la década de los años noventa, hoy estos mercados exhiben importantes disparidades en términos de sus niveles de desarrollo y capitalización, si se comparan con sus contrapartes de Estados Unidos y Canadá. La Tabla 1 presenta una descripción general del tamaño medido de las economías mediante su producto interno bruto (PIB) y mediante el nivel de capitalización de los mercados bursátiles y de bonos gubernamentales.

La Tabla 1 muestra que la economía de México es la más pequeña de la región del TLCAN. Su PIB registró un valor real de US \$1.026,4 billones en 2017. Dicho monto es aproximadamente 17 veces menor al monto registrado en el mismo año por la economía de Estados Unidos (US $\$ 17.305,0$ billones) y 1,4 veces menor al registrado por la economía de Canadá (US \$1.475,2 billones). Sin embargo, debe destacarse que la economía mexicana presentó el mayor dinamismo durante el período analizado, al registrar una tasa de crecimiento real promedio del PIB de 3,32 por ciento. La economía de Estados Unidos, por su parte, tuvo el menor dinamismo, ya que su tasa de crecimiento real promedio fue de 2,28 por ciento.

Las diferencias en el tamaño de las economías también se reflejan en sus mercados financieros. Como puede observarse en la misma tabla, los niveles de capitalización de los mercados de Estados Unidos y Canadá son mucho más altos que los registrados por los mercados de México. Al finalizar el año 2017 la capitalización del mercado bursátil de Estados Unidos y Canadá medido como proporción respecto a su PIB fue de 165,7 y 143,2 por ciento respectivamente, mientras que el de México fue de apenas 36,3 por ciento. En términos absolutos, la capitalización del mercado bursátil de Estados Unidos al cierre del mismo año fue aproximadamente 14 veces mayor que la de Canadá y 77 veces mayor que la de México.

En los mercados de bonos gubernamentales se observan las mismas asimetrías. En el 2010, año para el cual se encontró la información más reciente, el nivel de capitalización del mercado de Estados Unidos registró un valor de US $\$ 1.788$ billones, el cual es aproximadamente 10 veces mayor 
al nivel de capitalización del mercado de Canadá (US\$167,7 billones) y 57 veces mayor al de México (US $\$ 31,2$ billones).

Tabla 1. Producto interno bruto y nivel de capitalización de los mercados financieros

\begin{tabular}{|c|c|c|c|c|c|c|c|}
\hline Indicador & 1995 & 2000 & 2005 & 2010 & 2015 & 2017 & $\begin{array}{l}\text { Tasa de } \\
\text { crecimiento } \\
\text { promedio }\end{array}$ \\
\hline \multicolumn{8}{|c|}{ Producto interno bruto (Billones de USD) } \\
\hline Estados Unidos & $10.299,0$ & $12.713,1$ & $14.408,1$ & $14.964,4$ & $16.672,7$ & $17.305,0$ & 2,28 \\
\hline Canadá & 811,7 & 917,5 & $1.286,8$ & $1.613,5$ & $1.435,0$ & $1.475,2$ & 2,63 \\
\hline México & 483,9 & 875,1 & 965,6 & $1.057,8$ & $1.076,2$ & $1.026,4$ & 3,32 \\
\hline \multicolumn{8}{|c|}{ Capitalización del mercado bursátil (Billones de USD) } \\
\hline Estados Unidos & $9.342,2$ & $18.674,8$ & $18.707,4$ & $17.283,5$ & $23.064,4$ & $28.665,9$ & 5,00 \\
\hline Canadá & 902,7 & 952,8 & $1.631,0$ & $2.171,2$ & $1.466,1$ & $2.112,5$ & 3,77 \\
\hline México & 121,9 & 154,8 & 263,1 & 454,3 & 370,1 & 372,2 & 4,97 \\
\hline \multicolumn{8}{|c|}{ Capitalización del mercado bursátil/PIB ( \%) } \\
\hline Estados Unidos & 90,7 & 146,9 & 129,8 & 115,5 & 138,3 & 165,7 & 2,65 \\
\hline Canadá & 111,2 & 103,8 & 126,8 & 134,6 & 102,2 & 143,2 & 1,11 \\
\hline México & 25,2 & 17,7 & 27,3 & 43,0 & 34,4 & 36,3 & 1,60 \\
\hline \multicolumn{8}{|c|}{ Capitalización del mercado de valores gubernamentales (Billones de USD) } \\
\hline Estados Unidos & 997,8 & 761,7 & $1.006,0$ & $1.788,5$ & & & 3,71 \\
\hline Canadá & 156,8 & 65,5 & 115,4 & 167,6 & & & 0,42 \\
\hline México & 9,23 & 22,00 & 29,10 & 31,18 & & & 7,90 \\
\hline
\end{tabular}

Nota: los valores presentados son contabilizados en dólares americanos a precios constantes de 2010. Los valores sobre el nivel de capitalización del mercado monetario se refieren únicamente a la capitalización de los mercados de Treasury Bills y fueron obtenidos de la base de datos sobre Deuda del Gobierno Central publicada por la OCDE hasta el año 2010.

Fuente: elaboración propia con información estadística del Banco Mundial y la OCDE.

Las asimetrías entre los mercados financieros de los países que conforman el TLCAN también se reflejan en sus dinámicas. La Figura 1 presenta las gráficas de los doce mercados analizados. En ella se observan las diferencias entre las dinámicas que siguen los mercados de Estados Unidos y Canadá, y aquellas de los mercados de México. Resulta importante enfatizar que la información mostrada en dichas gráficas inicia en el año 1995, justo después de la crisis económica ocurrida en México en el año 1994, e incluyen otros eventos de crisis internacionales, entre estos la última crisis financiera global iniciada en 2007. 
Anguiano-Pita y Ruiz-Porras: Dinámicas e integración de los mercados financieros...

Figura 1. Dinámica de los mercados de valores gubernamentales, tipos de cambio e indices bursátiles de la región del TLCAN, 1995-2017
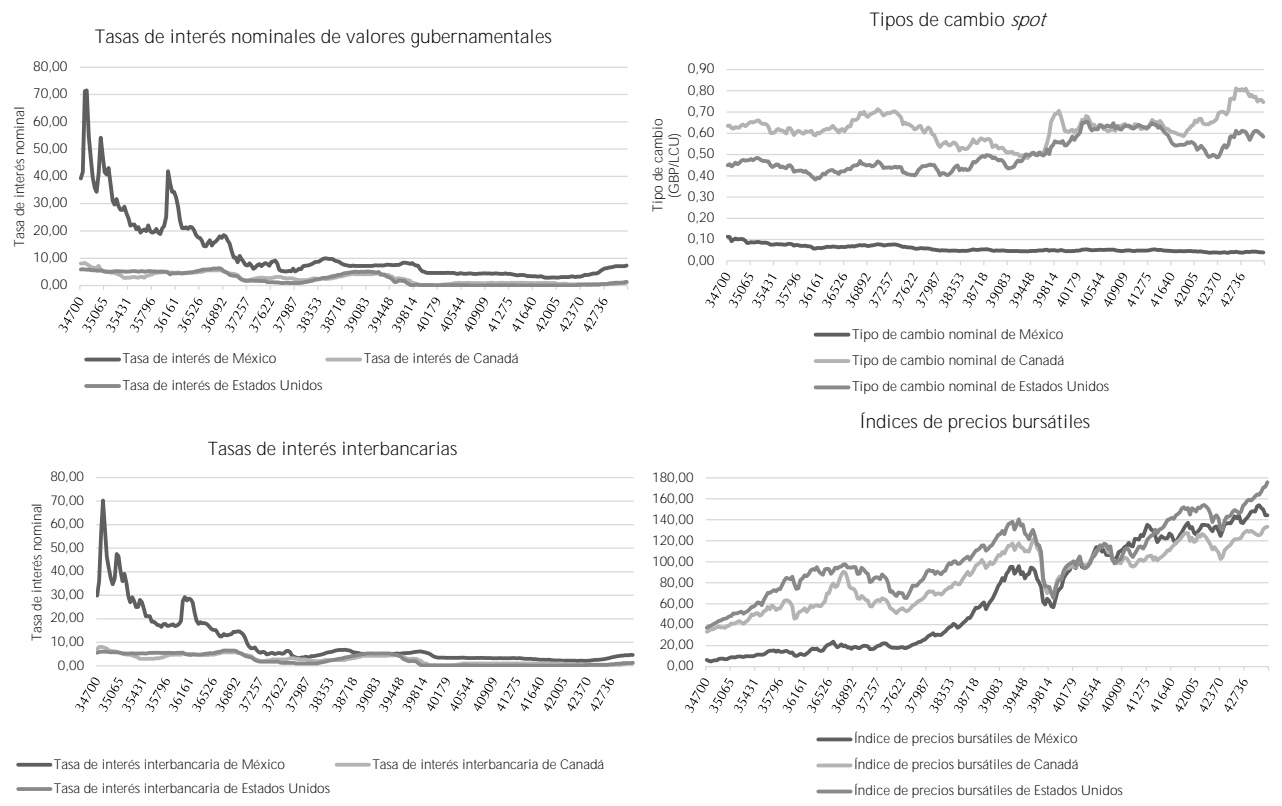

Nota: las variables consideradas corresponden a las series en niveles. Los tipos de cambio consideran el valor de las monedas de cada país (Local Currency Unit-LCU-) con respecto a la libra esterlina (GBP). Los índices de precios bursátiles consideran como período base el año 2010.

Fuente: elaboración propia.

La evidencia sugiere elementos importantes a considerar para el estudio y la caracterización de la integración financiera en la región del TLCAN. En primer lugar, resulta importante considerar las diferencias en los tamaños y niveles de desarrollo de los mercados, ya que esto puede ser una barrera que limite su plena integración. Por otra parte, mediante el análisis gráfico se puede inferir que el proceso de integración financiera regional tiene un carácter dinámico y que es posible que existan cambios estructurales en las series, debido a que el período analizado coincide con distintos eventos de crisis económicas y financieras. Particularmente, estas consideraciones motivan y validan el enfoque metodológico usado en esta investigación. 


\section{Revisión de la literatura}

El estudio de los diversos procesos de integración financiera a nivel mundial ha sido un tema de gran interés para la literatura empírica. En la literatura contemporánea es posible identificar diversos enfoques metodológicos e indicadores que han sido empleados para medir el grado de avance de la integración entre mercados. Sin embargo, debe señalarse que en esta literatura no existe un consenso sobre la definición de la noción de integración financiera ${ }^{1}$ ni tampoco sobre una técnica específica para cuantificar su avance. En este estudio se evalúa la hipótesis de Ho (2009), la cual argumenta que los acuerdos comerciales inducen la integración de los mercados financieros. ${ }^{2}$

En el contexto particular de la región del TLCAN, los estudios empíricos que han analizado la integración de los mercados financieros son escasos. Los estudios existentes suelen centrar su atención en los mercados bursátiles y tienden a concluir que el nivel de integración entre estos es moderado. Este hallazgo se explica por las diferencias en sus niveles de capitalización, eficiencia, nivel de riesgo y costos de transacción (Ortiz, López-Herrera \& Cabello, 2007; López-Herrera \& Ortiz, 2010).

La literatura que emplea el análisis de cointegración para estudiar los mercados financieros del TLCAN son los más comunes. Particularmente, Aggarwal y Kyaw (2005) y López-Herrera y Ortiz (2010) argumentan que

1 En la literatura especializada se encuentran distintas definiciones relacionadas con el concepto de integración. Eyraud, Singh y Sutton (2017, p. 5) la definen como "el proceso mediante el cual los mercados de dos o más países, o regiones, se vuelven más entrelazados entre sî” y con los grandes centros financieros internacionales. Por otra parte, Sutherland (1996) describe el concepto como un proceso en el cual los mercados convergen en términos de coberturas de activos y se enfrentan a los mismos shocks debido a la existencia de mecanismos y canales de contagio y propagación de crisis.

2 Ho (2009) explica que la integración financiera ocurre debido a la entrada en vigor de acuerdos formales e informales entre países. En este contexto, los acuerdos formales se definen como aquellos en los que se pacta la armonización o eliminación de las regulaciones que restringen la entrada de los agentes e intermediarios de los países miembros. Los acuerdos informales, por su parte, son aquellos que permiten a las empresas nacionales y extranjeras compartir su información, invertir de manera conjunta o gestionar préstamos. 
Anguiano-Pita y Ruiz-Porras: Dinámicas e integración de los mercados financieros...

el nivel de integración de los mercados bursátiles se ha incrementado desde la entrada en vigor del acuerdo comercial. Ortiz, López-Herrera y Cabello (2007) y López-Herrera, Ortiz y Cabello (2009) señalan la importancia de considerar cambios estructurales en la integración entre los mercados. RuizPorras y Anguiano-Pita (2017a) sugieren que la integración entre los mercados monetarios, cambiarios y de bienes y servicios ha sido limitada. ${ }^{3}$

La literatura que emplea el enfoque de volatilidades y correlaciones condicionales mediante variantes de los modelos GARCH (modelos generalizados auto regresivos condicionalmente heterocedásticos) ha encontrado que el nivel de interrelaciones e interdependencias entre los mercados bursátiles es moderado, pero tiende a aumentar durante momentos de crisis (p. ej. Chambet \& Gibson, 2008; Lahrech \& Sylwester, 2013; Sosa \& Ortiz, 2017). Por el contrario, Ruiz-Porras y Anguiano-Pita (2017b) presentan resultados divergentes, pues señalan que los mercados monetarios, cambiarios, y de bienes y servicios presentan bajos niveles de integración; además, resaltan que estos responden de forma asimétrica ante shocks de información diferenciados.

Un enfoque relativamente poco empleado por la literatura que ha estudiado previamente los mercados del TLCAN es el análisis de factores. López-Herrera (2006) y López-Herrera y Ortiz (2011) estiman modelos multifactoriales para evaluar la dinámica de la prima de riesgo del mercado de capitales de México e identificar las relaciones entre los factores macroeconómicos locales y el proceso de determinación de precios en los mercados bursátiles de México y la región del TLCAN. El principal hallazgo de estos estudios señala que el nivel de integración entre los mercados es moderado debido a que predomina la influencia de los factores locales sobre los internacionales.

3 El estudio de López-Herrera et al. (2009) emplea el análisis de cointegración para analizar las relaciones de largo plazo entre los mercados bursátiles del TLCAN, y posteriormente evalúa el corto plazo mediante el estudio de las interrelaciones e interdependencias entre mercados. Por otra parte, el estudio de Ruiz-Porras y Anguiano-Pita (2017a) evalúa la existencia de relaciones de largo plazo entre los mercados monetarios, cambiarios, y de bienes y servicios para, posteriormente, analizar la dinámica de corto plazo de los mercados mediante pruebas de causalidad de Granger. 
Las limitaciones de los estudios empíricos que han analizado la integración financiera en el TLCAN son áreas de oportunidad en la agenda de investigación regional. El análisis de cointegración se sustenta en que las series analizadas son integradas de orden uno - I(1) - , homocedásticas y que se distribuyen normalmente. Estos supuestos resultan restrictivos cuando se estudian series con cambios estructurales. El análisis de volatilidades y correlaciones condicionales mediante modelos GARCH ve limitada su estimación e interpretación cuando se estudia un número relativamente grande de activos financieros.

En los últimos años han surgido distintos enfoques metodológicos que pretenden añadir robustez a los indicadores empleados para estudiar la integración financiera. Los modelos de factores constituyen una posible área de oportunidad para la literatura empírica. Este enfoque de modelación permite analizar las dinámicas comunes de comportamiento de grandes paneles de series de tiempo. Sus principales ventajas suponen análisis más precisos, modelos más parsimoniosos y mayor flexibilidad, ya que no se tiene que incurrir en supuestos sobre la relación teórica entre las variables analizadas.

La literatura que emplea modelos de factores dinámicos para estudiar la integración financiera es relativamente reciente. Cipriani y Kaminski (2006) analizan los patrones de integración de los mercados bursátiles, de bonos y de préstamos sindicados de 101 países. Este estudio emplea un modelo bayesiano de factor dinámico no estacionario e interpreta la varianza explicada por los factores comunes como medida de integración. Sus principales hallazgos señalan mayores similitudes en la dinámica de integración de los mercados de bonos y de emisión de préstamos sindicados, en comparación con la de los mercados bursátiles.

Qin, Cagas, Ducanes, Magtibay-Ramos y Quising (2007) utilizan un enfoque de modelación que combina la estimación de factores dinámicos y un modelo de corrección de errores para estudiar el proceso de integración de doce economías asiáticas. El estudio se sustenta en el análisis de las condiciones internacionales de paridad. Los principales hallazgos del estudio señalan una mayor velocidad e intensidad en los niveles de integración entre 
Anguiano-Pita y Ruiz-Porras: Dinámicas e integración de los mercados financieros...

los mercados de bienes en comparación con los mercados de tasas de interés y tipos de cambio.

Existen estudios que analizan la integración de mercados específicos. De Paula-Rocha y Marino-Sekkel (2006) estudian la integración de veinticuatro mercados bursátiles de economías desarrolladas y emergentes, utilizando el enfoque generalizado de factores dinámicos. Los principales resultados de este estudio señalan mayores niveles de integración entre los mercados de economías desarrolladas. En un contexto local, Baele, Bekaert e Ingelbrecht (2010) estudian los co-movimientos entre los rendimientos de los mercados de bonos y bursátiles de Estados Unidos. Sus principales hallazgos destacan la limitada contribución de la información macroeconómica para explicar las correlaciones entre mercados.

Boysen-Hegrefe (2013) emplea un modelo de factores dinámicos que considera volatilidades estocásticas en los componentes idiosincráticos y parámetros que cambian en el tiempo; esto, para estudiar la dinámica de los mercados de bonos gubernamentales de la Unión Europea. Sus principales hallazgos señalan un proceso de desintegración entre los mercados de países que han enfrentado crisis de deuda. Por otra parte, Greenaway-McGrevy, Mark, Sul y Wu (2018) estudian los rendimientos de los mercados de tipos de cambio de veintisiete países. Sus resultados señalan que los mercados de tipos de cambio son conducidos por dos factores empíricos vinculados con el dólar americano y el euro.

La principal limitación de los estudios empíricos estriba en la falta de consenso entre los enfoques teóricos que explican la integración entre mercados financieros. En este sentido, el estudio desarrollado aquí complementa la literatura existente porque incluye distintos mercados financieros sin suponer una relación única entre estos. Más aún, este modelo de análisis permite evitar el planteamiento de supuestos a priori sobre las relaciones entre las variables o sobre los canales específicos que ha seguido el proceso de integración financiera de la región del TLCAN. La existencia de un proceso de integración se justifica con base en la presencia de factores comunes entre las series analizadas. 
La pertinencia de usar los modelos de factores dinámicos se justifica porque no hay razones para suponer que los factores que definen el proceso de integración sean únicamente estáticos. En este estudio se emplea el modelo generalizado de factores dinámicos propuesto por Forni et al. (2005). Este enfoque permite sintetizar las similitudes en la dinámica de las series estudiadas, sin imponer una forma funcional específica; además, aprovecha toda la información contenida en las series observadas en todo el espectro de frecuencias, lo que permite capturar los movimientos coincidentes de corto y largo plazo. Esta última característica resulta importante si se considera la naturaleza dinámica de los procesos de integración económica y financiera.

Finalmente, es importante señalar que la revisión de la literatura evidencia la conveniencia de estudiar la integración de los mercados financieros de la región del TLCAN. En primer lugar, esta investigación es importante por la falta de estudios sobre la integración de mercados financieros distintos a los bursátiles; asimismo, por la naturaleza dinámica del proceso de integración. Esta investigación también se relaciona con el actual proceso de modernización y los posibles resultados de la renegociación del tratado comercial. Estas consideraciones constituyen en sí mismas la motivación y validan el enfoque metodológico empleado.

\section{Metodología de análisis}

En este estudio se analizan las series mensuales de tasas de interés, tipos de cambio e índices de precios bursátiles de Estados Unidos, Canadá y México mediante un enfoque de series de tiempo. La metodología del estudio se sustenta en análisis de tipo estadístico, de cambio estructural y de componentes comunes. El análisis estadístico se usa para caracterizar las series y determinar su orden de integración, las pruebas de cambio estructural se emplean para determinar endógenamente la existencia de quiebres (p. ej. cambios estructurales) en las series, y el análisis de componentes comunes se emplea para evaluar el nivel de integración entre los mercados estudiados.

El análisis estadístico se sustenta en estadísticas descriptivas y en pruebas de normalidad, raíz unitaria y estacionariedad. Las estadísticas descriptivas y las pruebas de normalidad de Jarque-Bera se usan para caracterizar la 
Anguiano-Pita y Ruiz-Porras: Dinámicas e integración de los mercados financieros...

dinámica del comportamiento de las series de rendimientos, mientras que las pruebas de raíz unitaria y estacionariedad se emplean para determinar el orden de integración de las series. ${ }^{4}$ En este estudio se emplean las pruebas de raíz unitaria aumentada de Dickey-Fuller (ADF) y de estacionariedad de Kwiatkowski-Phillips-Schmidt-Shin (KPSS) sobre las series en niveles y en diferencias de cada variable ${ }^{5}$ ambas se hacen haciendo diferentes supuestos para determinar el orden de integración de las series de manera robusta.

El análisis de cambio estructural endógeno complementa al análisis de orden de integración. En este análisis se emplea la prueba de Zivot-Andrews (ZA) para evaluar endógenamente la potencial existencia de un quiebre (p. ej. un cambio estructural) en una serie considerada como I(1). Esta prueba se estima repetidamente sobre las series en niveles para determinar el momento de ocurrencia de un posible cambio estructural. La hipótesis nula asociada a esta prueba es que la serie tiene una raíz unitaria y que no experimenta ningún quiebre. La hipótesis alternativa es que la serie es estacionaria y que experimenta un quiebre en un período en específico.

Econométricamente, este estudio se sustenta en la estimación del modelo generalizado de factores dinámicos propuesto por Forni et al. (2005). Previo a su estimación, la metodología requiere la selección del número de factores estáticos y dinámicos. En este estudio se emplean los criterios de información de Alessi et al. (2010) y Hallin y Liska (2007) para determinar el número de factores que minimizan la varianza de los componentes idiosincráticos. Se seleccionan ambos criterios de información para llegar a resultados robustos y porque los mismos tienen un buen desempeño en el análisis de muestras finitas.

El análisis de componentes comunes sirve para evaluar el nivel de integración entre los mercados financieros con base en la propuesta de Forni et al. (2000), la cual sostiene que el cociente entre la varianza del componente

4 La correcta identificación del orden de integración de las series analizadas es importante para evitar la modelación de correlaciones espurias.

5 Se emplean ambas pruebas para contrastar las hipótesis nulas asociadas a cada una de ellas. La hipótesis nula de la prueba $\mathrm{ADF}$ es que la serie analizada tiene una raíz unitaria; en tanto que la hipótesis alternativa es que la serie es estacionaria, $\mathrm{I}(0)$. Por el contrario, la hipótesis nula de la prueba KPSS es que la serie analizada es estacionaria. 
común y la varianza total de cada variable observada es una medida del nivel de comunalidad de cada variable en el sistema. En el marco de este estudio, la medida resultante puede ser interpretada como un índice sintético de la integración entre los mercados analizados. En este sentido, se estiman correlaciones de Pearson para establecer el grado de asociación entre los componentes comunes y las variables observadas.

El enfoque de modelación empleado en este estudio tiene algunas ventajas con respecto a otros enfoques econométricos, pues con este es posible estudiar la dinámica de un conjunto de series sin imponer una relación teórica específica entre ellas. Esto es especialmente útil para estudiar la relación entre diferentes mercados financieros. Adicionalmente, el enfoque usado permite la modelación conjunta de los componentes comunes aun cuando las series manifiestan cambios estructurales. 6 Finalmente, el enfoque empleado aprovecha la información contenida en las series analizadas en todos los espectros de frecuencia, lo que permite capturar de forma más precisa los movimientos coincidentes de corto y largo plazo. ${ }^{7}$

Metodológicamente, el modelo de factores propuesto por Forni et al. (2005) supone que un conjunto de $N$ vectores de series de tiempo puede ser representado como la suma de dos componentes mutuamente ortogonales: un componente común dirigido por un número reducido de $q<N$ factores comunes y un componente idiosincrático relacionado específicamente a cada $N$ variable.

Supóngase que $X_{i t}$ es una vector de dimensión $(T \times N)$ que contiene $T$ observaciones de cada una de las $N$ series de tiempo estacionarias con media 0 y covarianza $\Gamma(0)$. Si dicho vector es un modelo generalizado de factores dinámicos es posible demostrar que cada serie de tiempo puede ser representada mediante la ecuación $(1):^{8}$

6 Adviértase que las pruebas tradicionales de cointegración requieren que las series no manifiesten quiebres, cambios estructurales. Este requisito hace inviable estudiar la integración de largo plazo con las pruebas mencionadas.

7 Sala (2001) muestra que el análisis de cointegración visto desde el dominio de frecuencias es equivalente al estudio de la densidad espectral en la frecuencia cero.

8 Las condiciones necesarias y los supuestos que debe satisfacer el modelo se encuentran en Forni et al. (2000, 2005). 
Anguiano-Pita y Ruiz-Porras: Dinámicas e integración de los mercados financieros...

$$
X_{i t}=\chi_{i t}+\xi_{i t}=\sum_{j=1}^{q} b_{i j}(L) u_{j t}+\xi_{j t},
$$

donde $\chi_{i t}$ es el componente común y $\xi_{j t}$ el componente idiosincrático. En este contexto, $b_{i j}(L)=B_{n}(L)=B_{0}^{n}+B_{1}^{n} L+\cdots+B_{s}^{n} L^{s}$ representa las ponderaciones dinámicas de carga de orden $s$, a las cuales se les permite que difieran en coeficiente y rezagos entre las series. Los $q$ factores o shocks comunes $\left(u_{j t} ; j=1, \ldots, q ; t \in Z\right)$, asociados a $B_{n}(L)$, se suponen como procesos de ruido blanco con varianza unitaria mutuamente ortogonales en todos sus rezagos y adelantos. ${ }^{9}$ El componente idiosincrático es dirigido por shocks específicos de cada variable. La estructura del factor dinámico implica que el componente idiosincrático de todas las series es ortogonal al componente común en todos sus rezagos y adelantos.

Los factores comunes $u_{j t}$ son variables latentes que se estiman empleando la técnica de componentes principales dinámicos. Mediante esta técnica las variables observadas son desplazadas en el tiempo antes de ser promediadas entre las unidades de sección cruzada, tomando en cuenta el conjunto completo de covarianzas dinámicas. La idea detrás de este método supone que, al promediar entre unidades de sección cruzada y desplazando las series en el tiempo, los componentes idiosincráticos débilmente correlacionados se cancelan, mientras que las fuentes de variación común se conservan.

La matriz de densidad espectral $\Sigma_{n}(\theta)=\left(\sigma_{i j}(\theta)\right)$ de $X_{i t}$ es estimada utilizando el análisis espectral de series de tiempo. Para cada frecuencia $[-\pi<\theta<\pi]$, se obtienen los componentes principales dinámicos a través de la descomposición de la matriz de densidad espectral en sus respectivos vectores propios y valores propios. Los componentes comunes son las proyecciones ortogonales de las series observadas en el presente, pasado y futuro de los primeros $q$ componentes principales dinámicos. Los componentes idiosincráticos pueden ser encontrados después de sustraer el componente común a las series observadas.

9 El vector $B_{n}(L)$ tiene una matriz de densidad espectral no-singular, equivalente a los primeros $q$ valores propios dinámicos del conjunto de información analizada. 
Finalmente, debe enfatizarse que la metodología propuesta en este estudio tiene cuatro objetivos: primero, caracterizar las series de tasas de interés, tipos de cambio e índices de precios bursátiles; segundo, determinar endógenamente si han existido cambios estructurales en dichas variables; tercero, evaluar la existencia de factores comunes que señalen algún tipo de integración entre los mercados estudiados; y, por último, calcular el porcentaje de la varianza total explicada por los componentes comunes. Estos objetivos son importantes para comprender algunos aspectos del proceso de integración financiera en la región del TLCAN.

\section{Base de datos y estadística descriptiva}

La base de datos empleada en este estudio se compone por un panel de series mensuales de tasas de interés, tipos de cambio spot e índices de precios bursátiles representativos de los mercados de Estados Unidos, Canadá y México para el período entre enero de 1995 y diciembre de 2017. Cada una de las series abarca un total de 276 observaciones. ${ }^{10}$

Para construir los rendimientos de los mercados de tipos de cambio y bursátiles se emplean las primeras diferencias de las series en logaritmos. Las series de rendimientos de los mercados de tasas de interés se expresan de manera anualizada. Específicamente, las tasas de interés analizadas son representativas de los mercados de valores gubernamentales e interbancarios. Los mercados de valores gubernamentales son caracterizados por las tasas de interés pagadas por bonos gubernamentales con período de madurez constante de tres meses. ${ }^{11}$ Los mercados interbancarios son caracterizados por las tasas de interés interbancarias menores a 24 horas (Call Money) Interbank interest rate) publicadas por la OCDE.

10 La selección del marco temporal obedece a dos razones principales: primero, la existencia de un régimen de tipo de cambio controlado en México prevaleciente hasta el 21 de diciembre de 1994; segundo, la información de buena calidad representativa del mercado de valores gubernamentales de México se encuentra disponible a partir del año 1995.

11 En el caso de Estados Unidos y Canadá se consideran las tasas de interés pagadas por las Letras del Tesoro (Treasury Bills). Para México se considera la tasa de interés pagada por los Certificados de la Tesorería de la Federación (CETES). 
Anguiano-Pita y Ruiz-Porras: Dinámicas e integración de los mercados financieros...

Para lograr un análisis consistente, los tipos de cambio se expresan en términos de la cotización spot al cierre de la moneda de cada país con respecto a la libra esterlina. Finalmente, el mercado bursátil de Estados Unidos es caracterizado mediante el índice bursátil New York Stock Exchange (NYSE), el de Canadá por el índice Toronto Stock Exchange (TSX), y el de México por el Índice de Precios y Cotizaciones (IPC). Las series de índices de precios bursátiles consideran el año 2010 como período base. La Tabla 2 presenta un resumen de las variables empleadas en este estudio y la fuente de la cual fueron recuperadas.

Tabla 2. Series empleadas

\begin{tabular}{lcc}
\hline Nombre de la serie & Fuente & Periodicidad \\
\hline Tasas de interés de valores gubernamentales & & Mensual \\
Letras del Tesoro a tres meses de Estados Unidos & Reserva Federal U.S. & Mensual \\
Letras del Tesoro a tres meses de Canadá & CANSIM Tabla 176-0043 & Mensual \\
Certificados de la Tesorería de la Federación a 91 días de México & Banco de México & Mensual \\
Tasas de interés interbancarias & OCDE & Mensual \\
Tasa de interés interbancaria de Estados Unidos & OCDE & Mensual \\
Tasa de interés interbancaria de Canadá & OCDE & Mensual \\
Tasa de interés interbancaria de México & & Mensual \\
Tipos de cambio & Reserva Federal U.S. & Mensual \\
Tipo de cambio spot de Estados Unidos & CANSIM Tabla 176-0064 & INEGI \\
Tipo de cambio spot de Canadá & & Mensual \\
Tipo de cambio spot de México & New York Stock Exchange & Mensual \\
Índices bursátiles & Standard \& Poor’s & Mensual \\
Índice bursátil de Estados Unidos & Bolsa Mexicana de Valores \\
Índice bursátil de Canadá & & \\
Índice bursátil de México &
\end{tabular}

Fuente: elaboración propia.

La Tabla 3 sintetiza la estadística descriptiva de las series de rendimientos durante el período analizado. En general, la tabla muestra que la totalidad de las series son leptocúrticas. Además, ocho series exhiben un sesgo negativo y cuatro positivo. De acuerdo con los estadísticos de Jarque-Bera y los valores $p$ calculados, la mayoría de las series no se distribuyen normalmente. 
Tabla 3. Estadística descriptiva de las series de rendimientos no estandarizados

\begin{tabular}{|c|c|c|c|c|c|c|c|}
\hline Nombre de la serie & Promedio & $\begin{array}{l}\text { Desviación } \\
\text { estándar }\end{array}$ & $\begin{array}{l}\text { Coeficiente } \\
\text { de asimetría }\end{array}$ & Curtosis & Jarque- Bera & Valor $\mathbf{p}$ & $\begin{array}{c}\text { Número de } \\
\text { observaciones }\end{array}$ \\
\hline \multicolumn{8}{|c|}{ Tasas de interés de valores gubernamentales } \\
\hline $\begin{array}{l}\text { Letras del Tesoro a tres meses } \\
\text { de Estados Unidos }\end{array}$ & 5,0537 & 48,6771 & 6,9014 & 67,8829 & $50.420,1700$ & 0,000 & 275 \\
\hline $\begin{array}{l}\text { Letras del Tesoro a tres meses } \\
\text { de Canadá }\end{array}$ & $-0,0205$ & 11,9052 & 0,8405 & 13,4662 & $1.287,5270$ & 0,000 & 275 \\
\hline $\begin{array}{l}\text { Certificados de la Tesorería de la } \\
\text { Federación a } 91 \text { días de México }\end{array}$ & $-0,2271$ & 9,2875 & 2,9490 & 24,3828 & $5.637,5980$ & 0,000 & 275 \\
\hline \multicolumn{8}{|l|}{ Tasas de interés interbancarias } \\
\hline $\begin{array}{l}\text { Tasa de interés interbancaria } \\
\text { de Estados Unidos }\end{array}$ & 0,3585 & 13,1311 & 1,1584 & 18,3960 & $2.777,5620$ & 0,000 & 275 \\
\hline $\begin{array}{l}\text { Tasa de interés interbancaria } \\
\text { de Canadá }\end{array}$ & $-0,1380$ & 11,0211 & 2,7742 & 35,5018 & $12.456,9000$ & 0,000 & 275 \\
\hline $\begin{array}{l}\text { Tasa de interés interbancaria } \\
\text { de México }\end{array}$ & $-0,4049$ & 7,6527 & 2,2502 & 19,7605 & $3.450,8860$ & 0,000 & 275 \\
\hline \multicolumn{8}{|l|}{ Tipos de cambio } \\
\hline $\begin{array}{l}\text { Tipo de cambio spot de Estados } \\
\text { Unidos }\end{array}$ & 0,0954 & 2,0772 & 0,1038 & 3,5566 & 4,0430 & 0,132 & 275 \\
\hline Tipo de cambio spot de Canadá & 0,0586 & 2,0564 & 0,5866 & 5,3220 & 77,5528 & 0,000 & 275 \\
\hline Tipo de cambio spot de México & $-0,3887$ & 3,2684 & $-0,9327$ & 7,1377 & 236,0430 & 0,000 & 275 \\
\hline \multicolumn{8}{|l|}{ Índices bursátiles } \\
\hline $\begin{array}{l}\text { Índice bursátil de Estados } \\
\text { Unidos }\end{array}$ & 0,5659 & 3,7511 & $-1,6780$ & 11,6452 & 985,4416 & 0,000 & 275 \\
\hline Índice bursátil de Canadá & 0,5048 & 4,0450 & $-1,6905$ & 11,5501 & 968,6489 & 0,000 & 275 \\
\hline Índice bursátil de México & 1,1339 & 5,6850 & $-0,6362$ & 5,0405 & 66,2593 & 0,000 & 275 \\
\hline
\end{tabular}

Nota: las series de rendimientos de las tasas de interés de valores gubernamentales e interbancarias fueron calculadas a partir de tasas de crecimiento simples. Las series de rendimientos de tipos de cambio e índices de precios bursátiles fueron calculadas a partir de las primeras diferencias de las series en logaritmos.

Fuente: elaboración propia.

En la misma tabla se observa que el promedio de los rendimientos de la tasa de interés pagada por los valores gubernamentales de Estados Unidos y de los tres mercados bursátiles han sido los más altos, mientras que los rendimientos de los mercados de tasas de interés de Canadá y México han sido negativos. Asimismo, las desviaciones estándar muestran que los rendimientos de los mercados cambiarios de los tres países han sido relativamente más 
Anguiano-Pita y Ruiz-Porras: Dinámicas e integración de los mercados financieros...

estables en comparación con los demás mercados estudiados. Por último, el promedio de los rendimientos de los mercados cambiarios sugiere que las monedas de Estados Unidos y Canadá se han apreciado, mientras que la moneda de México se ha depreciado.

El modelo econométrico empleado en este estudio requiere que las variables sean estacionarias. Para evaluar el orden de integración de las series se emplean las pruebas de raíz unitaria ADF, de estacionariedad de KPSS y de cambio estructural endógeno de Zivot-Andrews. Las estimaciones de las pruebas de raíz unitaria y estacionariedad se presentan en la Tabla $1 \mathrm{~A}$ del anexo. Los resultados de las pruebas ADF indican que todas las series son estacionarias a partir de sus primeras diferencias. Por el contrario, los resultados de las pruebas KPSS indican que la tasa de interés interbancaria de Estados Unidos no cumple con el principio de estacionariedad, pues se consideran en términos de sus primeras diferencias. Asimismo, también se encontró evidencia de cambios estructurales, específicamente en la tasa de interés de valores gubernamentales de México y las tasas de interés interbancarias de México y Canadá (véase Tabla 4). Con la finalidad de asegurar la estacionariedad de las series analizadas, se optó por considerar únicamente sus rendimientos, ya que resultan ser estacionarias al menos en una de las pruebas implementadas.

Los resultados de esta sección pueden sintetizarse de la siguiente manera: los rendimientos de la tasa de interés pagada por los valores gubernamentales de Estados Unidos y de los tres mercados bursátiles han sido más altos en comparación con los mercados interbancarios y de tipos de cambio. Durante el período analizado, los rendimientos de los mercados de tasas de interés han sido los más volátiles. El tipo de cambio de México ha tendido a depreciarse, mientras que el de Estados Unidos y Canadá se ha apreciado. Finalmente, los resultados muestran que existe evidencia de posibles cambios estructurales en las series analizadas. 
Tabla 4. Pruebas de cambio estructural endógeno de Zivot-Andrews

\begin{tabular}{|c|c|c|c|c|c|c|}
\hline \multirow{2}{*}{ Nombre de la serie } & \multicolumn{2}{|c|}{ Quiebre en intercepto } & \multicolumn{2}{|c|}{ Quiebre en tendencia } & \multicolumn{2}{|c|}{ Quiebre en intercepto y tendencia } \\
\hline & Fecha & Significancia & Fecha & Significancia & Fecha & Significancia \\
\hline \multicolumn{7}{|c|}{ Tasas de interés de valores gubernamentales } \\
\hline $\begin{array}{l}\text { Letras del Tesoro a tres meses } \\
\text { de Estados Unidos }\end{array}$ & $2000 \mathrm{M} 12$ & & 2014M2 & & 2008M7 & \\
\hline $\begin{array}{l}\text { Letras del Tesoro a tres meses } \\
\text { de Canadá }\end{array}$ & 2008M1 & & 2016M3 & & 2008M7 & \\
\hline $\begin{array}{l}\text { Certificados de la Tesorería de la } \\
\text { Federación a } 91 \text { días de México }\end{array}$ & 1999M2 & $* * *$ & 2002M5 & $* * *$ & $2001 \mathrm{M} 5$ & ** \\
\hline \multicolumn{7}{|l|}{ Tasas de interés interbancarias } \\
\hline $\begin{array}{l}\text { Tasa de interés interbancaria } \\
\text { de Estados Unidos }\end{array}$ & 2001M1 & & 2014M2 & & $2008 \mathrm{M} 10$ & \\
\hline $\begin{array}{l}\text { Tasa de interés interbancaria } \\
\text { de Canadá }\end{array}$ & $2008 \mathrm{M} 10$ & $* *$ & $2015 \mathrm{M} 8$ & * & $2008 \mathrm{M} 10$ & ** \\
\hline $\begin{array}{l}\text { Tasa de interés interbancaria } \\
\text { de México }\end{array}$ & 1999M3 & $* * *$ & $2002 \mathrm{M} 5$ & $* * *$ & $2001 \mathrm{M} 5$ & $* *$ \\
\hline \multicolumn{7}{|l|}{ Tipos de cambio } \\
\hline $\begin{array}{l}\text { Tipo de cambio spot de Estados } \\
\text { Unidos }\end{array}$ & 2003M9 & & 2006M12 & & 2003M9 & \\
\hline Tipo de cambio spot de Canadá & $2007 \mathrm{M} 5$ & & $1996 \mathrm{M} 12$ & & $2008 \mathrm{M} 11$ & \\
\hline Tipo de cambio spot de México & $2002 \mathrm{M} 5$ & & $1996 \mathrm{M} 5$ & & $2002 \mathrm{M} 5$ & \\
\hline \multicolumn{7}{|l|}{ Índices bursátiles } \\
\hline Índice bursátil de Estados Unidos & 2008M6 & & $1997 \mathrm{M} 6$ & & $2008 \mathrm{M} 6$ & \\
\hline Índice bursátil de Canadá & $2008 \mathrm{M} 7$ & & 2006M9 & & $2008 \mathrm{M} 7$ & \\
\hline Índice bursátil de México & $2004 \mathrm{M} 9$ & & 2010M4 & & $2005 \mathrm{M} 5$ & \\
\hline
\end{tabular}

Nota: las pruebas se estimaron utilizando las series en niveles. El número de asteriscos $\left({ }^{*},{ }^{* *} \mathrm{y}{ }^{* * *}\right)$ denota el nivel de significancia estadística al 10, 5 y 1 por ciento, respectivamente.

Fuente: elaboración propia.

\section{Modelación y análisis econométrico}

La estimación del modelo de factores dinámicos requiere determinar los factores comunes entre las series estudiadas. En el presente estudio se emplean los criterios de información de Alessi et al. (2010) y Hallin y Liska (2007) para identificar respectivamente el número de factores estáticos y 
Anguiano-Pita y Ruiz-Porras: Dinámicas e integración de los mercados financieros...

dinámicos empleando las doce series de rendimientos estandarizados que son representativas de los mercados financieros de la región del TLCAN. En la Figura 2 se presentan las gráficas que ilustran los resultados de los criterios de información previamente mencionados.

Figura 2. Criterios de información para determinar el número óptimo de factores de las series
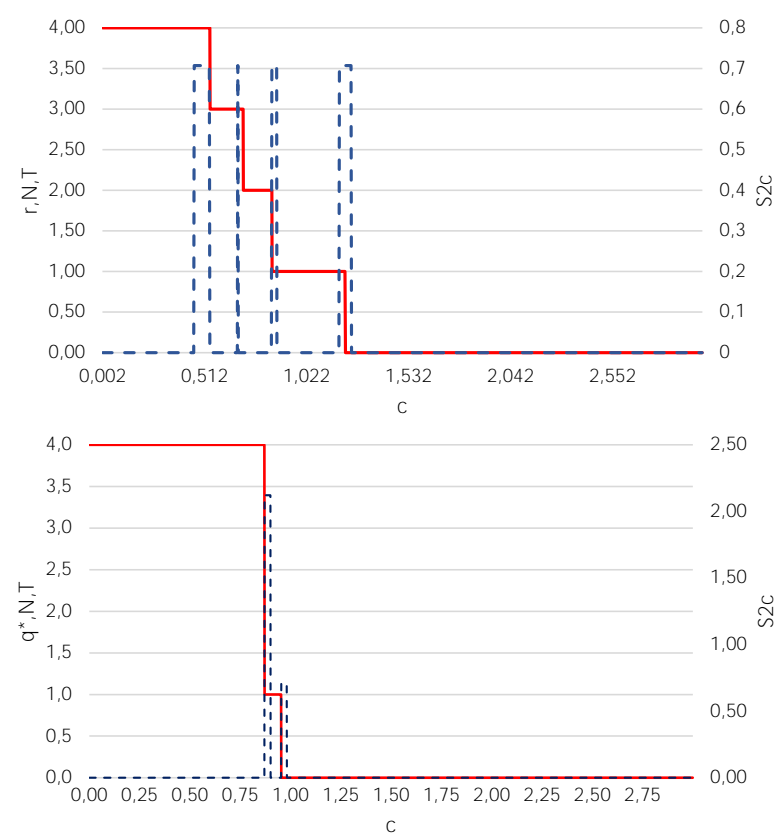

Nota: el límite superior de factores estáticos y dinámicos se fijó arbitrariamente en $r_{\text {máx }}=4$. El valor de la constante empleada para evaluar la función de penalización considera $c=3$. El parámetro de truncamiento de rezagos y de puntos en donde se evalúa la densidad espectral se fijó en $M=h=\sqrt{T}=16$.

Fuente: elaboración propia.

De acuerdo con los resultados presentados en el panel (a) de la Figura 2, el número óptimo de factores estáticos a retener es de tres, mientras que el panel (b) señala que el número de factores dinámicos óptimo es uno. Forni, Giannoni, Lippi y Reichlin (2009) señalan que las diferencias en el número óptimo de factores estáticos y dinámicos puede deberse a la heterogeneidad de 
las respuestas dinámicas de los componentes comunes a los shocks primitivos ${ }^{12}$. En el contexto de este estudio, los resultados encontrados sugieren que los mercados estudiados responden de manera heterogénea a las perturbaciones.

El modelo generalizado de factores dinámicos se estimó considerando tres factores estáticos y un factor dinámico. ${ }^{13}$ Con la finalidad de evaluar el nivel de integración entre los mercados financieros de la región del TLCAN, se estimaron los componentes comunes e idiosincráticos asociados a cada variable observada. En la Figura 1A del anexo se grafican las series de los componentes idiosincráticos estimadas y se presentan las estimaciones de los estadísticos ADF vinculadas a dichas series. Los resultados muestran que las series son estacionarias, lo cual valida que la modelación de los comovimientos de las series de rendimientos fue realizada de manera adecuada (véase Sala, 2001).

Los componentes comunes estimados por el modelo generalizado de factores dinámicos y las series de rendimientos estandarizadas se presentan en la Figura 3. El análisis de los coeficientes de correlación de Pearson $(\rho)$ y los valores $p$ contenidos en dicha figura sugiere que los componentes comunes parecen adecuarse en buena medida a la dinámica de los rendimientos de los mercados estudiados. En términos generales, los mayores coeficientes de correlación se observan en los mercados de tipos de cambio y bursátiles.

Siguiendo la propuesta de Forni et al. (2000) se calculó el porcentaje de la varianza total explicada por el componente común asociado a cada variable observada. Asimismo, se calcularon los promedios de los porcentajes y se agruparon por mercado y por país para analizar sus similitudes y diferencias. Los resultados se presentan en la Tabla 5.

12 Dentro de la literatura econométrica de factores dinámicos se entienden como choques dinámicos, primitivos o fundamentales a los choques estructurales que constituyen el origen de las fluctuaciones comunes a todas las variables estudiadas.

13 Las estimaciones econométricas se realizaron en Matlab R2018a. Los códigos de los procedimientos para la estimación del modelo generalizado de factores dinámicos fueron recuperados de la página personal de Matteo Barigozzi (http://www.barigozzi.eu/Codes.html) y adecuados a los propósitos del presente estudio. 
Anguiano-Pita y Ruiz-Porras: Dinámicas e integración de los mercados financieros...

Figura 3. Series de rendimientos observadas y componentes comunes estimados

Panel A. Rendimientos de las tasas de interés de valores gubernamentales

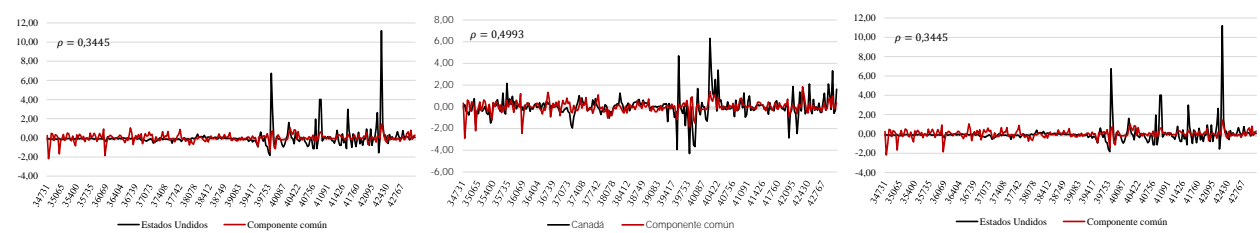

Panel B. Rendimientos de las tasas de interés interbancarias
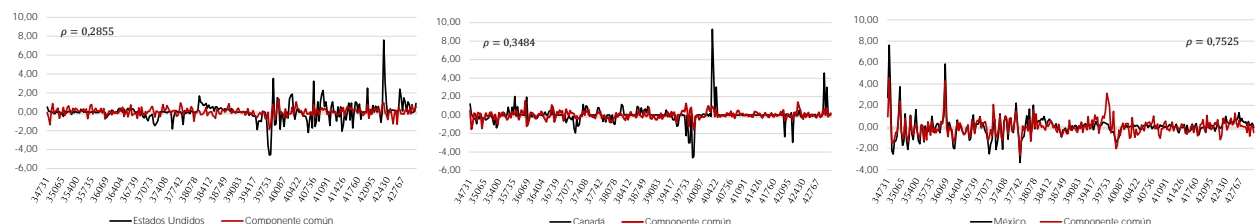

Panel C. Rendimientos de los tipos de cambio

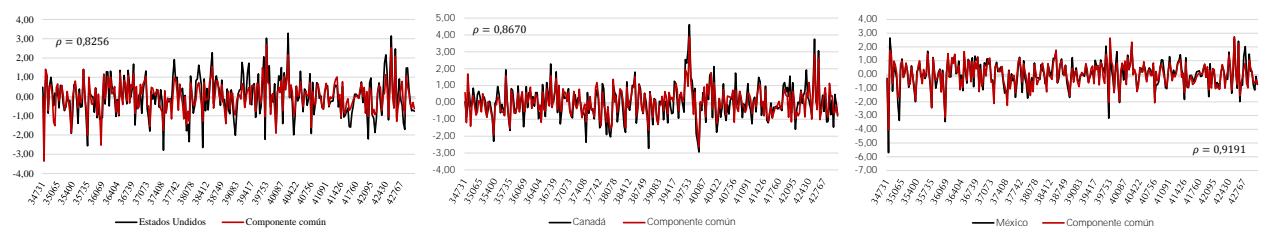

Panel D. Rendimientos de los indices bursátiles
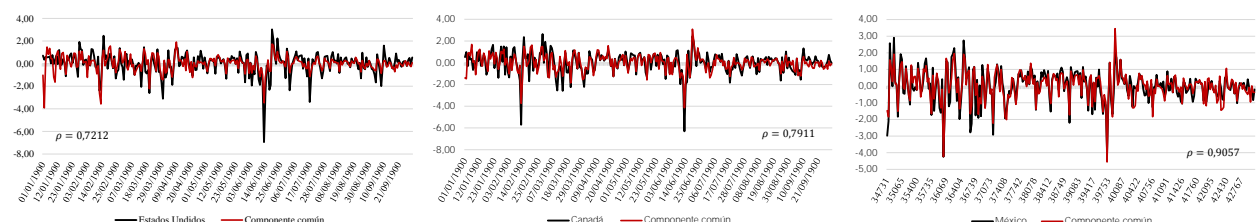

Nota: los componentes comunes fueron obtenidos a partir de la estimación del modelo generalizado de factores dinámicos empleando series de rendimientos estandarizados. El valor de $\rho$ se refiere al coeficiente de correlación de Pearson calculado para las series de rendimientos y de componentes comunes. El valor entre paréntesis se refiere al valor $\mathrm{p}$ asociado a cada estadístico.

Fuente: elaboración propia.

La Tabla 5 muestra que los porcentajes de la varianza total explicada por los componentes comunes varía entre mercados y países. En promedio, el 
69,10 por ciento de la varianza de los mercados cambiarios de la región se puede explicar por los componentes comunes, 66,45 por ciento en el caso de los mercados bursátiles, 35,16 por ciento en el caso de los mercados interbancarios y 34,50 por ciento en el caso de los mercados de valores gubernamentales. Los mayores porcentajes observados en los mercados cambiarios y bursátiles sugieren un mayor nivel de integración regional entre estos.

Tabla 5. Porcentaje de la varianza explicada por los componentes comunes

\begin{tabular}{lcccc}
\hline País & Estados Unidos & Canadá & México & $\begin{array}{c}\text { Promedio por } \\
\text { mercado }\end{array}$ \\
\hline Mercado de valores gubernamentales & 16,99 & 28,89 & 57,62 & 34,50 \\
Mercado interbancario & 17,86 & 18,63 & 68,98 & 35,16 \\
Mercado cambiario & 60,39 & 64,95 & 81,95 & 69,10 \\
Mercado bursátil & 81,38 & 64,19 & 53,77 & 66,45 \\
\hline Promedio por país & $\mathbf{4 4 , 1 5}$ & $\mathbf{4 4 , 1 7}$ & $\mathbf{6 5 , 5 8}$ & $\mathbf{5 1 , 3 0}$ \\
\hline
\end{tabular}

Nota: el cálculo de los porcentajes de la varianza explicada por los componentes comunes considera series de rendimientos estandarizados.

Fuente: elaboración propia.

Haciendo un análisis desagregado se observa que los mercados de tasas de interés de Estados Unidos y Canadá responden principalmente a sus componentes idiosincráticos. De acuerdo con la tabla anterior, los componentes comunes de ambos países explican únicamente el 16,99 y 28,89 por ciento de la varianza de los mercados de valores gubernamentales, y 17,86 y 18,63 por ciento de la varianza de los mercados interbancarios. Por el contrario, la influencia de los componentes comunes predomina en el mercado cambiario de México y el mercado bursátil de Estados Unidos, ya que los porcentajes de varianza explicada por estos es de 81,95 y 81,38 por ciento, respectivamente.

Finalmente, al hacer un análisis de los promedios de los porcentajes de la varianza explicada por los componentes comunes por país, se encontró que, en el caso de los mercados de México, dicho promedio fue de 65,58 por ciento, mientras que para Estados Unidos y Canadá fue de 44,15 y 44,17 
Anguiano-Pita y Ruiz-Porras: Dinámicas e integración de los mercados financieros...

por ciento, respectivamente. Las diferencias encontradas suponen que las dinámicas de los rendimientos de los mercados financieros de México tienen una mayor sensibilidad a las fluctuaciones de sus contrapartes en Estados Unidos y Canadá, pero esta condición no se cumpliría a la inversa.

Los resultados encontrados implican que la diversificación entre los mercados de valores gubernamentales e interbancarios de la región del TLCAN es una opción para los inversionistas. La distribución de portafolios de inversión entre los distintos mercados financieros de la región puede disminuir en cierta medida la exposición al riesgo de los inversionistas, ya que la dinámica de dichos mercados exhibe respuestas proporcionalmente distintas a los choques comunes al interior de la región.

\section{Conclusiones}

En esta investigación se han estudiado las dinámicas y el nivel de integración entre los mercados financieros de los países que conforman la región del TLCAN. El estudio se sustenta en la estimación del modelo generalizado de factor dinámico propuesto por Forni et al. (2005). De esta manera se han estudiado: primero, la dinámica de las series de rendimientos de tasas de interés, tipos de cambio y de precios bursátiles; segundo, la existencia de cambios estructurales en las series; tercero, la existencia de factores comunes, y, finalmente el nivel de integración entre los mercados estudiados.

El estudio ha utilizado estadísticas descriptivas, pruebas de raíz unitaria, estacionariedad, de cambio estructural endógeno y la modelación econométrica de factores dinámicos. La base de datos analizada se compone por un panel de doce series de tiempo representativas de los mercados de tasas de interés de valores gubernamentales e interbancarias, tipos de cambio spot e índices de precios bursátiles de Estados Unidos, Canadá y México para el período comprendido entre enero de 1995 y diciembre de 2017.

Los principales hallazgos de este estudio demuestran la existencia de asimetrías en las características y las dinámicas de las series de rendimientos financieros. Se encontró evidencia de posibles cambios estructurales en las 
series de tasas de interés de valores gubernamentales de México y en las series de tasas de interés interbancarias de México y Canadá. Para evaluar el nivel de integración entre mercados financieros se calcularon los porcentajes de la varianza total de las series observadas explicada por los componentes comunes, obtenidos del modelo generalizado de factores dinámicos.

Los resultados señalan que los mercados estudiados distan de estar plenamente integrados. Se encontraron niveles más altos de integración entre los mercados cambiarios y bursátiles de los tres países. Por el contrario, los niveles de integración más bajos se encontraron entre los mercados de valores gubernamentales e interbancarios. Haciendo un análisis similar por país se encontró que las series de rendimientos de los mercados de México son más sensibles a los componentes comunes en comparación con aquellas de Estados Unidos y Canadá.

Los resultados encontrados en este estudio señalan que el nivel de integración financiera en la región del TLCAN es moderado, debido a la existencia de algunas ineficiencias que proveen oportunidades de diversificación y arbitraje para los inversionistas internacionales. Estas conclusiones resultan ser relevantes en el contexto de la modernización del acuerdo comercial y para la formulación de políticas económicas y financieras. El bajo nivel de integración entre los rendimientos de los mercados de valores gubernamentales e interbancarios justifica su utilidad como instrumentos de política monetaria en el corto plazo, durante momentos de incertidumbre y volatilidad entre los mercados de la región.

\section{Anexos}

\section{Determinación del orden de integración de las series}

A continuación, se presentan los resultados de las estimaciones de las pruebas de raíz unitaria ADF y de estacionariedad KPSS de las series empleadas en este estudio. Ambas pruebas fueron estimadas considerando una tendencia lineal y un intercepto determinista como regresores exógenos. En el caso de las pruebas ADF el número de rezagos fue seleccionado empleando el criterio de información de Schwartz-Bayesiano. La hipótesis 
Anguiano-Pita y Ruiz-Porras: Dinámicas e integración de los mercados financieros...

nula asociada a la prueba ADF se rechaza cuando el valor de la probabilidad es inferior al 5 por ciento. Por otra parte, la hipótesis nula asociada a la prueba KPSS se rechaza cuando el estadístico calculado es mayor al valor crítico asociado a la prueba. El valor crítico asociado a la prueba, considerando un nivel de significancia estadística del 5 por ciento es 0,1463 .

Tabla 1A. Pruebas de raiz unitaria ADF y de estacionariedad KPSS de las series estudiadas

\begin{tabular}{|c|c|c|c|c|c|c|c|c|}
\hline \multirow{3}{*}{ Nombre de la serie } & \multicolumn{4}{|c|}{ Pruebas ADF } & \multicolumn{4}{|c|}{ Pruebas KPSS } \\
\hline & \multicolumn{2}{|c|}{ Niveles } & \multicolumn{2}{|c|}{ Diferencias } & \multicolumn{2}{|c|}{ Niveles } & \multicolumn{2}{|c|}{ Diferencias } \\
\hline & Prob. & Rezagos & Prob. & Rezagos & Estadístico & Estacionariedad & Prob. & Estacionariedad \\
\hline \multicolumn{9}{|c|}{ Tasas de interés de valores gubernamentales } \\
\hline $\begin{array}{l}\text { Letras del Tesoro a Tres } \\
\text { Meses de Estados Unidos }\end{array}$ & 0,4850 & 3 & 0,000 & 2 & 0,0791 & * & 0,0490 & * \\
\hline $\begin{array}{l}\text { Letras del Tesoro a Tres } \\
\text { Meses de Canadá }\end{array}$ & 0,0604 & 3 & 0,000 & 0 & 0,0555 & * & 0,0327 & * \\
\hline $\begin{array}{l}\text { Certificados de la Tesorería } \\
\text { de la Federación a } 91 \text { días de } \\
\text { México }\end{array}$ & 0,0095 & 1 & 0,000 & 0 & 0,3705 & & 0,0553 & * \\
\hline \multicolumn{9}{|l|}{ Tasas de interés interbancarias } \\
\hline $\begin{array}{l}\text { Tasa de interés interbancaria } \\
\text { de Estados Unidos }\end{array}$ & 0,7267 & 1 & 0,000 & 0 & 0,0760 & * & 0,1562 & \\
\hline $\begin{array}{l}\text { Tasa de interés interbancaria } \\
\text { de Canadá }\end{array}$ & 0,0067 & 3 & 0,000 & 2 & 0,0487 & * & 0,0322 & * \\
\hline $\begin{array}{l}\text { Tasa de interés interbancaria } \\
\text { de México }\end{array}$ & 0,0308 & 6 & 0,000 & 0 & 0,3793 & & 0,0627 & * \\
\hline \multicolumn{9}{|l|}{ Tipos de cambio } \\
\hline $\begin{array}{l}\text { Tipo de cambio spot } \\
\text { de Estados Unidos }\end{array}$ & 0,6269 & 1 & 0,000 & 0 & 0,2689 & & 0,0411 & * \\
\hline $\begin{array}{l}\text { Tipo de cambio spot } \\
\text { de Canadá }\end{array}$ & 0,4937 & 1 & 0,000 & 0 & 0,1841 & & 0,0672 & * \\
\hline $\begin{array}{l}\text { Tipo de cambio spot } \\
\text { de México }\end{array}$ & 0,0544 & 0 & 0,000 & 0 & 0,2292 & & 0,0654 & * \\
\hline \multicolumn{9}{|l|}{ Índices bursátiles } \\
\hline $\begin{array}{l}\text { Índice bursátil de Estados } \\
\text { Unidos }\end{array}$ & 0,1129 & 1 & 0,000 & 0 & 0,1169 & * & 0,0894 & * \\
\hline Índice bursátil de Canadá & 0,0634 & 1 & 0,000 & 0 & 0,1515 & & 0,0402 & * \\
\hline Índice bursátil de México & 0,4536 & 1 & 0,000 & 0 & 0,2712 & & 0,0429 & * \\
\hline
\end{tabular}

Fuente: elaboración propia. 
Figura 1A. Componentes idiosincráticos estimados

Panel A. Componentes idiosincráticos estimados para los rendimientos de las tasas de valores gubernamentales

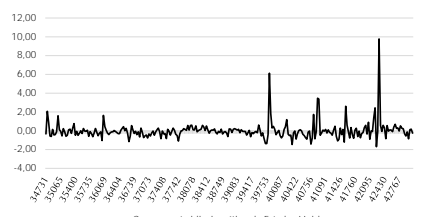

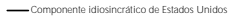

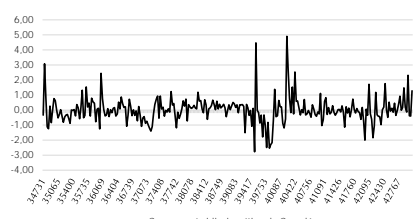

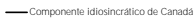

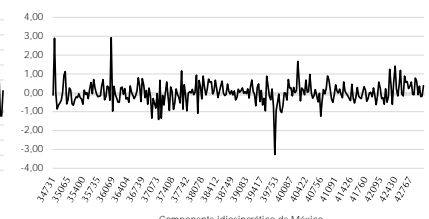

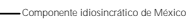

Panel B. Componentes idiosincráticos estimados para los rendimientos de las tasas de interés interbancarias

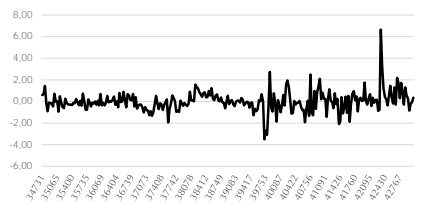

一

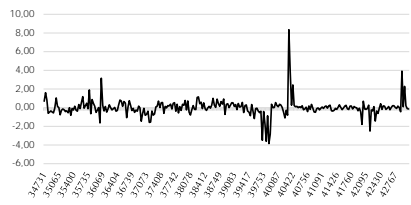

—Componente didsinur de cond

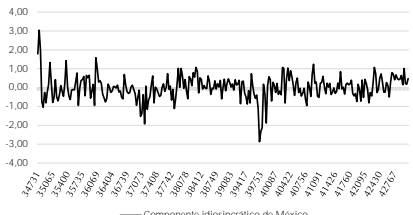

Panel C. Componentes idiosincráticos estimados para los rendimientos de los tipos de cambio spot
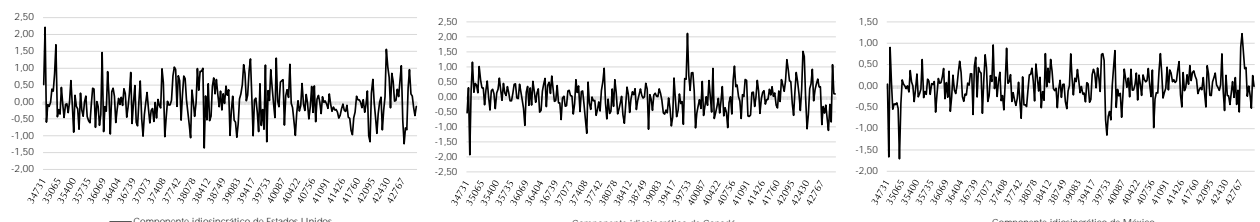

Panel D. Componentes idiosincráticos estimados para los rendimientos de los indices de precios bursátiles
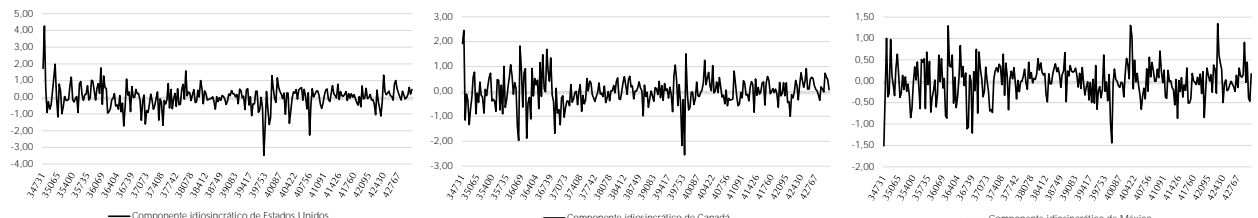

Nota: la estimación del modelo de factores dinámicos considera series de rendimientos estandarizadas. Fuente: elaboración propia. 
Anguiano-Pita y Ruiz-Porras: Dinámicas e integración de los mercados financieros...

En la Tabla 2A, se presentan las pruebas de raíz unitaria ADF y de estacionariedad KPSS de los componentes idiosincráticos estimados por el modelo generalizado de factores dinámicos. Ambas pruebas consideran una tendencia lineal y un intercepto determinista como regresores exógenos. En el caso de las pruebas ADF el número de rezagos fue seleccionado empleando el criterio de información de Schwartz-Bayesiano. La hipótesis nula asociada a la prueba $\mathrm{ADF}$ se rechaza cuando el valor de la probabilidad es inferior al 5 por ciento. Por otra parte, la hipótesis nula asociada a la prueba KPSS se rechaza cuando el estadístico calculado es mayor al valor crítico asociado a la prueba. El valor crítico asociado a la prueba, considerando un nivel de significancia estadística del 5 por ciento es 0,1463 .

Tabla 2A. Pruebas de raíz unitaria y de estacionariedad de los componentes idiosincráticos

\begin{tabular}{|c|c|c|c|c|c|c|c|c|}
\hline \multirow{3}{*}{ Nombre de la serie } & \multicolumn{4}{|c|}{ Pruebas ADF } & \multicolumn{4}{|c|}{ Pruebas KPSS } \\
\hline & \multicolumn{2}{|c|}{ Niveles } & \multicolumn{2}{|c|}{ Diferencias } & \multicolumn{2}{|c|}{ Niveles } & \multicolumn{2}{|c|}{ Diferencias } \\
\hline & Prob. & Rezagos & Prob. & Rezagos & Estadístico & Estacionariedad & Prob. & Estacionariedad \\
\hline \multicolumn{9}{|c|}{ Tasas de interés de valores gubernamentales } \\
\hline Estados Unidos & 0,0000 & 2 & 0,000 & 6 & 0,0532 & * & 0,1091 & * \\
\hline Canadá & 0,0000 & 0 & 0,000 & 4 & 0,0367 & * & 0,0986 & * \\
\hline México & 0,0000 & 0 & 0,000 & 3 & 0,0723 & * & 0,1252 & * \\
\hline \multicolumn{9}{|c|}{ Tasas de interés interbancarias } \\
\hline Estados Unidos & 0,0000 & 0 & 0,000 & 4 & 0,1338 & * & $0,0657^{*}$ & \\
\hline Canadá & 0,0000 & 2 & 0,000 & 1 & 0,0342 & $*$ & 0,1522 & \\
\hline México & 0,0000 & 0 & 0,000 & 3 & 0,1027 & * & 0,0562 & * \\
\hline \multicolumn{9}{|l|}{ Tipos de cambio } \\
\hline Estados Unidos & 0,0000 & 0 & 0,000 & 2 & 0,0716 & * & 0,3823 & \\
\hline Canadá & 0,0000 & 0 & 0,000 & 3 & 0,1281 & * & 0,0235 & * \\
\hline México & 0,0000 & 0 & 0,000 & 5 & 0,0564 & * & 0,0689 & $*$ \\
\hline \multicolumn{9}{|l|}{ Índices bursátiles } \\
\hline Estados Unidos & 0,0000 & 0 & 0,000 & 3 & 0,1117 & $*$ & 0,0148 & $*$ \\
\hline Canadá & 0,0000 & 0 & 0,000 & 6 & 0,0704 & * & 0,1525 & \\
\hline México & 0,0000 & 0 & 0,000 & 6 & 0,0512 & $*$ & 0,1595 & \\
\hline
\end{tabular}

Fuente: elaboración propia. 


\section{Agradecimientos}

Los autores agradecen las observaciones y sugerencias de dos dictaminadores anónimos de la revista Lecturas de Economía.

\section{Referencias}

Aggarwal, R. \& Kyaw, N. A. (2005). Equity market integration in the NAFTA region: Evidence from unit root and cointegration tests. International Review of Financial Analysis, 14(4), 393-406.

Alessi, L., Barigozzi, M. \& Capasso, M. (2010). Improved penalization for determining the number of factors in approximate factor models. Statistics \& Probability Letters, 80(23-24), 1806-1813.

Baele, L., Bekaert, G. \& Inghelbrecht, K. (2010). The determinants of stock and bond return comovements. Review of Financial Studies, 23(6), 23742428 .

Boysen-Hogrefe, J. (2013). A dynamic factor model with time-varying loadings for euro area bond markets during the debt crisis. Economic Letters, 18(1), 50-54.

Chambet, A. \& Gibson, R. (2008). Financial integration, economic instability and trade structure in emerging markets. Journal of International Money and Finance, 27(4), 654-675.

Cipriani, M. \& Kaminsky, G. (2006). A new era of international financial integration: global, market and regional factors (Economics \& finance workshop discussion paper series No. 534). Recuperado de ResearchGate: https://www.researchgate.net/publication/228684671_A_new_era_o f_international_financial_integration_global_market_and_regional_fac tors

De Paula-Rocha, B. \& Marino-Sekkel, R. (2006). The dynamic factor model: an application to international stock market integration. Revista de Economía. Segunda Época, XIII(1), 93-115. 
Anguiano-Pita y Ruiz-Porras: Dinámicas e integración de los mercados financieros...

Eiteman, D., Stonehill, A. \& Moffett, M. (2016). Multinational Business Finance. Harlow: Pearson Education Limited.

Eyraud, L., Singh, D. \& Sutton, B. (2017). Benefits of global and regional financial integration in Latin America (Working paper No.17/1). Recuperado del sitio web del Fondo Monetario Internacional: https: //www.imf.org/en/Publications/WP/Issues/2017/01/18/Benefits-of -Global-and-Regional-Financial-Integration-in-Latin-America-44548

Forni, M., Giannoni, D., Lippi, M. \& Reichlin, L. (2009). Opening the Black Box: Structural Factor Models with Large Cross-Sections. Econometric Theory, 25(5), 1319- 1347.

Forni, M., Hallin, M., Lippi, M. \& Reichlin, L. (2000). The generalized dynamic-factor model: Identification and estimation. Review of Economics and Statistics, 82(4), 540-554.

Forni, M., Hallin, M., Lippi, M. \& Reichlin, L. (2005). The Generalized Dynamic Factor Model: One-Sided Estimation and Forecasting. Journal of the American Statistical Association, 100(471), 830-840.

Greenaway-Mcgrevy, R., Mark, N., Sul, D. \& Wu, J.-L. (2018). Identifying exchange rate common factors. International Economic Review, 59(4), 2193-2218.

Hallin, M. \& Liska, R. (2007). Determining the Number of Factors in the General Dynamic Factor Model. Journal of the American Statistical Association, 102(478), 603-617.

Ho, N. W. (2009). Financial Integration: Concepts and Impacts. Macao Monetary Research, 10(1), 69-84.

Lahrech, A. \& Sylwester, K. (2013). The impact of NAFTA on North American stock market linkages. The North American Journal of Economics and Finance, 25, 94-108.

López-Herrera, F. (2006). Riesgo sistemático en el mercado mexicano de capitales: un caso de segmentación parcial. Contaduría y Administración, 219, 86-113. 
López-Herrera, F. \& Ortiz, E. (2010). Cointegration trends among the NAFTA equity markets. Revista de Economía Mundial, 26, 155-176.

López-Herrera, F. \& Ortiz, E. (2011). Dynamic multibeta macroeconomic asset pricing model at NAFTA stock markets. International Journal of Economics and Finance, 3(1), 55-68.

López-Herrera, F., Ortiz, E. \& Cabello, A. (2009). Las interrelaciones de volatilidad y rendimientos entre los mercados de valores del TLCAN. Investigación Económica, 68(267), 83-113.

Ortiz, E., López-Herrera, F. \& Cabello, A. (2007). Las bolsas de valores en el área del TLCAN: un análisis a largo plazo. Problemas del desarrollo, 38(151), 37-61.

Qin, D., Cagas, M. A., Ducanes, G., Magtibay-Ramos, N. \& Quising, P. F. (2007). Measuring Reginal Market Integration in Developing Asia: A Dynamic Factor Error Correction Model (DF-ECM) Approach (Working Paper Series on Regional Economic Integration No. 8). Recuperado del sitio web del Asian Development Bank: https://www.adb.org/publicatio $\mathrm{ns} /$ measuring-regional-market-integration-developing-asia-dynamic-f actor-error-correction

Ruiz-Porras, A. \& Anguiano-Pita, J. E. (2017a). Cointegración, causalidad y cambio estructural en la región del TLCAN. En Tinoco Zermeño, M., Torres Preciado, V. \& Venegas Martínez, F. (Eds.), Desafios de la Economía Mexicana: El Sector Externo I (pp. 137-166). México: IPN.

Ruiz-Porras, A. \& Anguiano-Pita, J. E. (2017b). Modelación GARCH multivariada de las variaciones de tasas de interés, tipos de cambio y precios, un estudio para México, Canadá y Estados Unidos. En CastilloRamírez, C., Venegas-Martínez, F. \& López-Herrera, F. (Eds.), Modelado de Fenómenos Económicos y Financieros: Una visión contemporánea (pp. 331-358). México: UDLAP. Recuperado de: http://yuss.me/revistas /Libros/book2017aFVMn016.pdf

Sala, L. (2001). Monetary Transmission in the Euro Area: A Factor Model Approach. Recuperado de: ftp://ftp.igier.unibocconi.it/varie/semina rs/250302.pdf 
Anguiano-Pita y Ruiz-Porras: Dinámicas e integración de los mercados financieros...

Sosa, M. \& Ortiz, E. (2017). Global financial crisis volatility impact and contagion effect on NAFTA equity markets. Estocástica: Finanzas y Riesgo, 7(1), 67-88.

Sutherland, A. (1996). Financial market integration and macroeconomic volatility, Scandinavian Journal of Economics, 98(4), 521-539. 